LABORATOIRE DE RECHERCHES SUR LA PHYSIQUE DES PLASMAS FINANCE PAR LE FONDS NATIONAL SUISSE DE LA RECHERCHE SCIENTIFIQUE

ON THE DESIGN OF A ROTATING FIELD PINCHED DISCHARGE

E.S. Weibel

R. Keller 


\title{
ON THE DESIGN OF A ROTATING FIEID PINCHED DISCHARGE
}

\author{
E.S. Weibel \\ I. Keller
}

A b s t $r$ a c t

The dynamics of a rotating field pinch is described in terms of the macroscopic quantities temperature, pressure, plasma radius and magnetic field strength. The amplitude program necessary for maintaining the pinch is established and heating rates are given. The energy transfer between generator and plasma is examined and the condition for maximum efficiency is derived. These are the relations upon which the design of an experiment should be based. 


\section{INTRODUCTION}

A plasma column which is confined by a rotating magnetic field is heated at the same time by the current induced in the boundary layer. Thus the field amplitude must increase in time to balance the rising plasma pressure.

This report established a relation between the magnetic field amplitude and the plasma radius, on the basis of a simple model. The plasma is considered as uniform in density and temperature at all times. The rate of heating is calculated in accordance with the theory of the skin effect, both normal and anomalous 1).

Inertia and heat losses are neglected. The former because the plasma contracts only slowly, the latter because the rate of heating is far greater than any loss by radiation.

In this connection it must be pointed out, that by neglecting energy losses we obtain conservative estimates of generator performance. This is so because the main problem encountered in alternating field confinement is due to the rapid heating of the plasma which necessitates a very rapid increase in field strength, and hence in power. If energy is lost from the plasma its pressure will rise more slowly and the power requirements on the generator will be easier to satisfy. Thus we believe to be on the safe side with our power estimates.

Pressure balance and conservation of energy lead then to a first order differential equation with respect to time relating the field amplitude and the plasma radius. This equation can be integrated and yields an explicit description of the dynamics of the pinched discharge. One may, for instance, prescribe the initial plasma parameters and the plasma radius as a function of time. The analysis then

1) E.S. Weibel, The anomalous skin effect in a plasma LRP $18 / 65$. 
Gives the required field amplitude as a function of time, that is, the necessary amplitude program.

As a matter of great practical importance, the transfer of energy from a generator to the experiment is also considered. The generator is characterized by its internal resistance and by its open circuit voltage. The plasma offers an impedance that is mainly inductive with a small resistive component. To obtain an efficient power transfer it is clear that the plasma inductance must be combined with a capacity to form a resonant circuit.

The conditions for total energy transfer are well known for resonant circuits with constant elements and for the steady state. But the necessity of a programmed increase of the field amplitude precludes any steady state.

Under these conditions good energy transfer appears impossible. It is not generally realized that this need not be so. It is theoretically possible to obtain total energy transfer from a generator to a re sonant circuit even during a transient (of a particular form). In practice energy transfer from generator to the experiment of more than 90 per cent is possible during the type of transients that are useful for the production of a pinch. of this energy about half is stored in the circuit and the rest is transformed into thermal plasma energy.

This report does not intend to give a detailed description of the phenomena taking place in a plasma when it is confined by a rotating field. As the title implies it gives macroscopic relations which permit the design of an experiment. 
Consider a cylindrical plasma of electron density $\mathrm{n}$ and temperature $T$ which is confined by a magnetic field of the form $\sqrt{2} \hat{B}(t)$ sin $(\omega t)$. Let $\ell$ be the length, a the radius of the plasma column and designate the volume, total number of electrons and total energy by $s_{p}, \mathbb{N}$ and $W_{p}$ respectively. Then the following relations hold

$$
\begin{aligned}
& s_{\mathrm{p}}=\pi l \mathrm{a}^{2} \\
& \mathbb{N}=\pi l \mathrm{a}^{2} \mathrm{n} \\
& W_{\mathrm{p}}=f k T n s_{p}=f k T N
\end{aligned}
$$

where $f$ is the number of degrees of freedom per particle. The balance of pressure requires

$$
p=2 k T n=\frac{\hat{B}^{2}}{2 \mu_{0}}
$$

Conservation of energy requires

$$
\dot{W}_{p}=-\dot{p} \dot{p} \dot{p}_{p}
$$

where $Q$ is the rate of heating due to the alternating current in the plasma boundary layer.

These equations can be considered to hold if the quantities involved vary sufficiently slowly in time. It shall be assumed that this is always the case and the plasma properties are considered uniform throughout its volume.

The rate of heating can be calculated from the theory of the skin effect. For a plasma the theory of the normal skin effect applies only up to a certain temperature $\mathrm{T}$ which is a function of the density and the frequency. We shall consider the two limiting cases of normal 
and extreme anomalous skin effect which are characterized by the following inequalities :

$$
\lambda=\frac{u^{2} \omega_{p}^{2} \omega}{c^{2}|i \omega+\nu|^{3}} \quad\left\{\begin{array}{l}
\text { I nomal } \\
\text { anomalous }
\end{array}\right.
$$

where

$$
\begin{aligned}
& u^{2}=2 \mathrm{kT} / \mathrm{m} \\
& \omega_{p}^{2}=e^{2} \mathrm{n} / \varepsilon_{0} m \\
& \nu=\frac{\lg \Lambda}{93} \frac{e^{4} \mathrm{n}}{\varepsilon_{0}^{2} \mathrm{~m}^{1 / 2}(\mathrm{kT})^{3 / 2}}
\end{aligned}
$$

Ignoring details we treat $\lg \Lambda$ as a constant and set it equal to 8.

As the plasma is heated up it will pass continuously from the normal condition (regarding skin effect) to the anomalous condition. In the following sections a) and $b$ ) we shall assume for simplicity that the transition occurs abruptly. In the normal and the anomalous domain the simple limiting formulas will be used which are valid, strictly speaking, only for $\lambda \ll l$ and $\lambda \gg l$. The transition is assumed to take place where the surface resistance calculated according to the two formulas has the same value.

\section{a) First heating phase : Normal Skin Effect.}

The theory of the normal skin effect gives an expression for the surface resistance which can be used to obtain $Q$ :

$$
R=\left(\frac{\mu_{0} \omega}{2 \sigma}\right)^{1 / 2} \quad, \quad \sigma=\frac{93}{1 g \Lambda} \quad \frac{\varepsilon_{0}^{2}(k r)^{3 / 2}}{e^{2} m^{1 / 2}}
$$

2) I. Spitzer, Physics of fully ionized gases, Interscience 1962. Definition of $\Lambda$ on $\mathrm{p} .128$. 
Hence

$$
Q=2 \pi \operatorname{la}_{\mathrm{a}} \hat{\mathrm{B}}^{2} / \mu_{0}^{2}
$$

The analysis becomes most transparent if one introduces dimensionless ratios to express $a(t)$ and $\hat{B}(t)$

$$
a=\rho(t) a_{0} \quad \hat{B}=B(t) B_{0}
$$

The quantities $a_{0}$ and $B_{0}$ are arbitrary. We further choose reference values $T_{0}$ and $n_{0}$ which are not entirely arbitrary but must satisfy equations (1) and (3), that is

$$
\pi \mathrm{la}_{0}^{2} \mathrm{n}_{0}=\mathrm{N}
$$

and

$$
4 \mu_{0} k T_{0} n_{0}=B_{0}^{2}
$$

With this preparation equation (4) assumes the simple form

$$
\frac{d}{d t}\left(\rho^{2} \beta^{2}\right)+\frac{2}{f} \beta^{2} \frac{d}{d t}\left(\rho^{2}\right)=\frac{8}{f} \frac{x_{0}}{\mu_{0} a_{0}}\left(\frac{\beta}{\rho}\right)^{1 / 2}
$$

The symbol $\mathscr{X}_{0}$ designates $\mathcal{R}$ for $T=T_{0}$.

The differential equation (9) can be solved readily

$$
\rho^{5 / 2}=\frac{1}{t_{0}} \beta^{-\frac{5}{28}} \int_{t^{t}}^{5 / 2(1 / 8-3 / 5)} d t^{\prime}
$$

with

$$
t_{0}=\frac{2+f}{5} \cdot \frac{\mu_{0} a_{0}}{2 \mu_{0}}
$$

and

$$
8=(2+f) / f
$$


This equation could just as easily have been solved for $\beta(t)$ in terms of $\rho(t)$. A fortunate coincidence simplifies the solution even more for a plasma with $f=3$, that is $8=5 / 3$ :

$$
\beta=\frac{\left(t / t_{0}+1\right)^{2 / 3}}{S^{5 / 3}}
$$

where

$$
t_{0}=\frac{\mu_{0} a_{0}}{2 \boldsymbol{R}_{0}}
$$

In this last equation the integration constant $t^{*}$ has been chosen such that for $t=0, \beta=\rho=1$. Therefore the quantities with subscript zero such as $B_{0}$ have, here, the meaning of initial values. It is curious to note that $t_{0}$ is just the time required for a static magnetic field to diffuse into the plasma for the initial conditions.

The time for heating the plasma from $\mathbb{T}_{0}$ to $T$ becomes

$$
t=\frac{\mu_{0} a}{2 \boldsymbol{x}(\mathrm{T})}-\frac{\mu_{0} a_{0}}{2 \boldsymbol{\mu}\left(\mathrm{T}_{0}\right)}
$$

It will be seen that the expression $\mu_{0} a / 2 \mathcal{N}$ equals the time constant of the electrical circuit up to a constant factor of order of unity which depends on the geometry of the experiment. Equation (10) can also be written in the form

$$
\frac{2 R(T)}{\mu_{0} a}=\frac{1}{t+t_{0}}
$$

showing how $\mathcal{R}$ decreases with time. 
b) Second heating phase : Anomalous Skin Effect.

The analysis for the heating due to the anomalous skin effect runs quite similarly to the one of the preceeding section. One must use the surface resistance

$$
x=\frac{2}{3 \sqrt{3} \pi^{1 / 6}} \sqrt{\frac{\mu_{0}}{\varepsilon_{0}}}\left(\frac{u}{c}\right)^{1 / 3}\left(\frac{\omega}{\omega_{p}}\right)^{2 / 3}
$$

where $u$ and $\omega_{p}$ have been defined previously in equation (5) and (6).

Thus the power dissipated in the plasma surface becomes

$$
\begin{aligned}
Q & =2 \pi l a \not \hat{B}^{2} / \mu_{0}^{2} \\
& =2 \pi \mu_{0}^{-2} l a_{0} B_{0}^{2} \varkappa\left(T_{0}, n_{0}\right) \beta^{I / 3} S^{2}
\end{aligned}
$$

where $\beta$ and $\rho$ have the analogous meaning as before, equations (8), but $\mathrm{B}_{0}, \mathrm{~T}_{0}, \mathrm{n}_{0}$ and $\mathrm{a}_{0}$ describe now the plasma conditions at the beginning of the second heating phase.

By introducing (12) into (4) one obtains the differential equation relating $\beta$ and $\rho$

$(f+2) \beta^{-1 / 3} \frac{d}{d t}(1, g)-3 f \frac{d}{d t}\left(\beta^{-1 / 3}\right)=\frac{4 R\left(T_{0}, n_{0}\right)}{\mu_{0} a_{0}}$

This equation can be solved for $\beta$, assuming $\rho$ prescribed :

$\beta^{-1 / 3}=-\frac{4}{3 f} \frac{R_{0}}{\mu_{0} a_{0}} \rho^{\frac{f+2}{3 f}} \int_{t^{*}}^{t} \rho^{-\frac{f+2}{3 f}}\left(t^{\prime}\right) d t^{\prime}$ 
Since the initial contraction of the plasma can be made to occur during the first phase it suffices to examine (13) for constant $\rho=1$. Taking $f=3$ and requiring $\beta(0)=1$ one obtains

$$
\beta=\left(\frac{t_{0}}{t_{0}-t}\right)^{3}
$$

where

$$
t_{0}=\frac{2}{4} \frac{\mu_{0} a_{0}}{x_{0}}
$$

Thus the heating is explosive, reaching infinite temperature in a finite time and the magnetic amplitude must follow by increasing with time according to the inverse third power. The time required to heat a plasma from temperature $\mathrm{T}_{I}$ to $\mathrm{T}$ is simply

$$
t=\frac{2}{4} \mu_{0} a_{0}\left(\frac{1}{p_{0}}-\frac{1}{n}\right)
$$

Thus the resistance increases as the inverse first power of time

$$
\frac{4 R}{9 \mu_{0} a_{0}}=\frac{1}{t_{0}-t}
$$

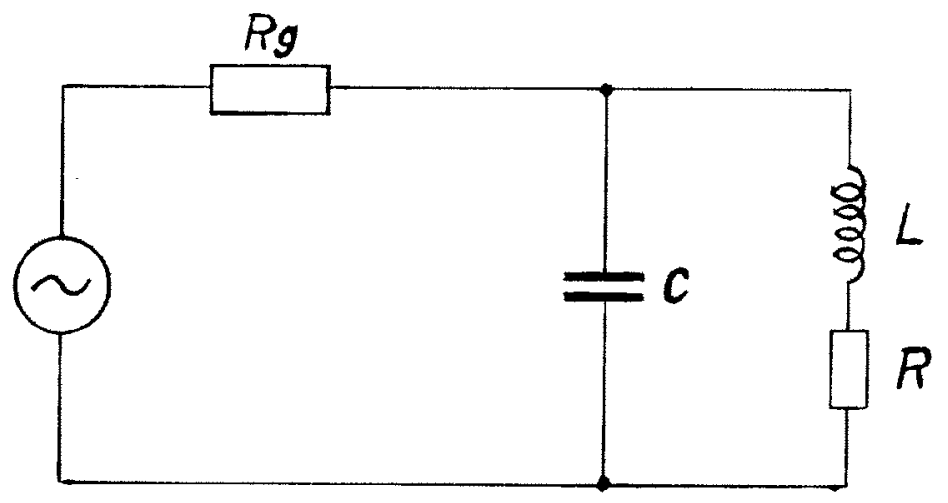

Fig. 3 
c) The standard amplitude promram, general case.

In sections a) and b) we treated the normal and anomalous case separately for the sake of simplicity. Thus the essential features of plasma heating and confinement could be clearly exhibited.

In reality, of course, there is a smooth transition between the two extreme cases. It so happens, that the surface resistance of the plasma is very accurately given by the sum of the normal and anomalous resistance. Thus

$$
n=n^{(\mathrm{n})}+n^{(\mathrm{a})}
$$

where $\boldsymbol{h}^{(\mathrm{n})}$ and $\boldsymbol{h}^{(\mathrm{a})}$ are given by the formulas (7) and (1l) respectively.

We now introduce new reference values $a_{0}, n_{0}, T_{0}, B_{0}$ in the following manner. Starting from a radius a, the plasma is assumed to contract initially to a radius $a_{0}$. Thereafter this radius is held constant. The density $n_{0}$ corresponds to this radius. $T_{0}$ is defined as the transition temperature associated with $n_{0}$, that is, the temperature at which $\mathcal{R}^{(\mathrm{n})}$ and $\mathcal{R}^{(\mathrm{a})}$ are equal. Finally $\mathrm{B}_{0}$ is chosen such that

$$
\mathrm{B}_{0}^{2}=4 \mu_{0} \mathrm{kT}_{0} \mathrm{n}_{0}
$$

Thus the pressure balance is satisfied for the reference values. Putting again

$$
a=\rho(t) a_{0}, \quad \hat{B}=\beta(t) B_{0}
$$

one can write the basic equation (4) in the form

$\frac{f+2}{f} \beta^{2} \rho \dot{\rho}+\dot{\beta \beta} \rho^{2}=\frac{v_{0}}{a_{0}}\left\{\beta^{1 / 2} \rho^{-1 / 2}+\beta^{7 / 3} \rho^{2}\right\}$ 
where

$$
v_{0}=\frac{4 \boldsymbol{x}_{0}}{3 \mu_{0}}
$$

and $\mathfrak{x}_{0}$ is the value of $\boldsymbol{x}^{(\mathrm{n})}=\boldsymbol{x}^{(\mathrm{a})}$ at the temperature $\mathrm{T}_{0}$. Unfortunately this last differential equation (14) is not even separable *).

We arrange the program such that the initial contraction takes place in the low temperature, normal regime. This allows us to use the simple explicit formulas of section a). The heating thereafter takes place at constant radius a,$\rho=l$. Integration of equ. (14) for $\rho=1$ gives

$$
\begin{aligned}
& t=\frac{a_{0}}{v_{0}} \tau(\beta) \\
& \tau=\int_{0}^{\beta} \frac{\alpha \beta}{\beta^{-1 / 2}+\beta^{4 / 3}}
\end{aligned}
$$

The integral represents, implicitly, an universal amplitude program $\beta(\tau)$ which has the same form in all possible cases except for changes in scale.

The heating power $Q$ can also be expressed in terms of a normalized function of $\beta$ :

$$
Q=\ell_{a} q_{0} p(\beta)
$$

where

$$
p=\beta^{1 / 2}+\beta^{7 / 3}
$$

*) Taking $x=\rho^{-\frac{5}{18}} \beta^{-1 / 6}$ this equation assumes the form $x=v_{0}\left(x^{10}+\rho^{-5 / 9} x^{-1}\right) / 6 a_{0}$, so that for $\rho=$ const. it can be integrated exactly, resulting in a complex sum of eleven logarithms. In practice it is much easier to integrate the original equation numerically. 
and

$$
q_{0}=\left(8 \pi \mathrm{k} / \mu_{0}\right) \mathrm{T}_{0} \mathrm{n}_{0} \boldsymbol{R}_{0}
$$

Fig. I represents graphically the reference values $a_{0}, B_{0}, v_{0}^{-1}$, $T_{0}$ as functions of $n_{0}$ and $f$, the frequency of the applied field. Fig. 2 shows $\tau$ and $p$ as functions of $\beta$.

\section{POWT TRANSFIR FROM GENERATOR TO PLASMA}

a) Circuit Equations.

Two circuits of the type shown in Fig. 3 are required to produce the rotating field : one for the $B_{z}$ and another for the $B_{\theta}$ component. The generator is assumed to have an internal resistance $\pi_{g}$ and develop the open circuit voltage

$$
V_{g}=\sqrt{2} \hat{V}_{g} \exp (i \omega t)
$$

during a finite time interval $0<t<t_{2}$. The maximum power that can be taken from this generator is

$$
\mathrm{P}_{\max }=\hat{\mathrm{V}}_{\mathrm{g}}^{2} / 4 \mathrm{R}_{\mathrm{g}}
$$

and the maximum energy during the entire pulse is

$$
W_{\text {max }}=\frac{1}{4 \pi_{g}} \int_{0}^{t_{g}} \hat{v}_{g}^{2}\left(t^{l}\right) d t^{2}
$$

The plasma is represented by the inductance $\tau$ and the resistance $\mathbb{R}$. The values of the circuit elements $I$ and $P$ are as follows : 
for the $\theta$ circuit :

$$
\begin{aligned}
& R=\frac{2 \pi a}{l} R \\
& I=\frac{\pi \mu_{0}}{l}\left(b^{2}-a^{2}\right) \\
& \frac{I}{\bar{R}}=\frac{1}{2}\left(b^{2} / a^{2}-1\right) \frac{\mu_{0} a}{n} \\
& B=\frac{\mu_{0} I}{l}
\end{aligned}
$$

for the $\mathbf{z}$ circuit :

$$
\begin{aligned}
& R=\frac{l}{2 \pi a} x \\
& I=\frac{\mu_{0}}{2 \pi} l \log b / a \\
& \frac{L}{\mathbb{R}}=\log (b / a) \frac{\mu_{0} a}{R} \\
& B=\frac{\mu_{0} I}{2 \pi a}
\end{aligned}
$$

In these formulas $\mathcal{R}$ is the surface impedance defined earlier for both normal and anomalous skin effect. The dimension b must be chosen such as to take account of all external self inductance of the circuit. $B$ is the magnetic field at the plasma surface in terms of the current in the circuit.

Taking into account the variation of the elements $\mathbb{R}$ and $I$, the equation for the current $I$ in the plasma assumes the form :

$\frac{V_{g}}{R_{g}}=\left(I+\frac{\dot{L}}{R_{g}}+\frac{R}{R_{g}}\right) I+\left(R C+\dot{L} C+\frac{L}{R_{g}}\right) \dot{I}+L C \ddot{I}$ 
One is really interested in the (rms) amplitude of the current. It is therefore convenient to derive an approximate equation of first order for this amplitude. By substituting

$$
I(t)=\sqrt{2} \hat{I}(t) e^{i \omega t}
$$

into equation (2I) and neglecting the terms $\mathrm{R} / \mathrm{L}, 1 / \mathrm{R}_{\mathrm{g}} \mathrm{C}$ and $\dot{\mathrm{L}} / \mathrm{L}$ against $\omega$ one finds the equation

$$
\left.\hat{V}_{g}=i 2 \omega \operatorname{LCR} g \frac{d}{d t} \hat{I}+(8-i \delta) \hat{I}\right\}
$$

where

$$
\begin{aligned}
& \gamma=\frac{1}{2}\left(\frac{\Gamma+\dot{L}}{L}+\frac{1}{\Pi_{g} C}\right) \\
& \delta=\frac{\omega}{2}\left[\left(1+\frac{\pi+\dot{L}}{R_{G}}\right) / \mathrm{LC} \omega^{2}-1\right]
\end{aligned}
$$

At resonance $\delta=0$. The pinch dynamics of chapter II leads to a prescription of $\hat{B}$ and hence of $\hat{I}$ as a function of t. Equation (22) now gives immediately the required generator voltage program.

b) Energy relations.

The total energy contained in the experiment is the sum of the plasma energy, $W_{p}$ and of the circuit energy $W_{c}$

$$
W=W_{p}+W_{c}
$$

where

$$
\begin{aligned}
& W_{p}=3 \mathbf{k T n s} p \\
& W_{c}=\hat{L I^{2}}=\frac{1}{\mu_{0}} \quad \hat{B}^{2} \dot{B}
\end{aligned}
$$


Equation (24) defines an equivalent magnetio volume, $s_{B}$, which is according to $(19.2),(19.4),(20.2)$ and $(20.4):$

$$
s_{B}= \begin{cases}2 \pi l a^{2} l g b / a & \text { for } z \text {-pinch } \\ \pi l\left(b^{2}-a^{2}\right) & \text { for } \theta \text {-pinch }\end{cases}
$$

As a consequence of the equilibrium of pressures (3) the ratio of plasma energy and circuit energy is only a function of the geometry of the experiment

$$
\frac{W_{p}}{W_{c}}=\frac{3 s_{p}}{4 s c_{B}}
$$

Consider now the power delivered to the experiment. It is the sum of the powers delivered to the resistor

$$
R I^{2}=Q
$$

to the capacitor

$$
\frac{d}{d t}\left(\frac{1}{2} C q^{2}\right)
$$

( $q$ being the charge of the capacitor) and to the inductor

$$
I \frac{d}{d t}(L I)=\frac{d}{d t}\left(\frac{I}{2} L I^{2}\right)+\frac{I}{2} \dot{L} I^{2}
$$

At resonance

$$
\begin{aligned}
& I=\sqrt{2} \hat{I}(t) \cos (\omega t), \\
& q=\sqrt{2} \hat{q}(t) \sin (\omega t),
\end{aligned}
$$

and

$$
C \hat{\mathrm{q}}^{2}=\mathrm{I} \hat{\mathrm{I}}^{2}
$$


By averaging over one period one obtains the total power delivered to the experiment

$$
P=\frac{d}{d t}\left(\hat{I}^{2}\right)+\left(\frac{I}{2} \dot{L}+I\right) \hat{I}^{2}
$$

The first term represents an increase of energy stored reversibly in the circuit as electric and magnetic fields, the second equals the mechanical work performed on the plasma by compression and the last term of course is the heating (27). It is perhaps useful to verify by independent deduction that

$$
\frac{1}{2} \dot{L I}^{2}
$$

is really the mechanical work.

To this end we first note that

$$
\frac{\dot{s} p}{s_{B}}=-\frac{\dot{I}}{I}
$$

for both $z$ - and $\theta$-circuits. Hence

$$
\frac{1}{2} \dot{L I} \hat{I}^{2}=\frac{\hat{L I}^{2} \dot{s} p}{2 S_{B}}
$$

Eliminating $\hat{I}^{2}$ with the help of $(24)$ and then replacing $\hat{B}^{2}$ by means of (3) gives indeed

$$
\frac{1}{2} \dot{L I} \hat{I}^{2}=-p \dot{p}
$$

The power delivered to the experiment (28) can now be expressed as follows

$$
\mathrm{P}=\dot{\mathrm{W}}_{\mathrm{c}}-\dot{\mathrm{p}} \dot{s}_{\mathrm{p}}+\mathrm{Q}
$$


The last two terms of this equation can be eliminated by means of equation (4) so that

$$
P=\dot{W}_{c}+\dot{w}_{p}
$$

This equation verifies that the energy delivered to the experiment is shared between the circuit and the plasma. Equation (26) shows in which proportion and permits one to eliminate $\nabla_{\mathrm{C}}$ :

$$
p=\left(1+\frac{4 s s_{B}}{35 s_{p}}\right) \dot{w}_{p}
$$

If the plasma radius remains constant as it should for most of the heating (except the initial contraction) then $\dot{s}_{\mathrm{p}}=0$ and

$$
P=\left(1+\frac{4 \Omega_{B}}{3 s_{p}}\right) Q
$$

By minimizing the stray inductances in the design of the experiment it is possible without too much difficulty to make si about equal to $s_{p}$ or even a little smaller. If one takes, as an example, $s_{B}=s_{p}$ then $P=2.33 \mathrm{Q}$. Thus the power which must be delivered to the experiment is considerably larger than the plasma heating power.

\section{c) Efficiency of Energy transfer}

Assume a prescribed program for $\hat{I}$ and hence for $\hat{V}_{g}$ such that $\dot{L}=0$ and that the circuit is always at resonance, $\delta=0$. Then the maximum available power from the generator is according to (18) and (22)

$$
\mathrm{P}_{\max }=\operatorname{LCR}_{g}\left(\dot{\hat{I}}^{2}+28 \hat{\mathrm{I}} \dot{\hat{I}}+8^{2} \hat{I}^{2}\right)
$$

while the power actually delivered to the experiment is

$$
P=2 \hat{L} \hat{I} \dot{I}+R \hat{I}^{2}
$$


A measure of the efficiency of energy transfer is the expression

$$
\frac{P_{\max }-P}{P}=\left(\frac{\dot{\hat{I}}+8 \hat{I}}{\dot{\hat{I}}+8 \hat{I}}\right)^{2}
$$

with

$$
\delta^{\prime}=\frac{1}{2}\left(\frac{R}{L}-\frac{1}{R_{g} C}\right)
$$

This last equation shows as would be expected that $P \leqslant P_{\max }$. One might not have expected, however, that total energy transfer is possible, in principle, even for a transient, albeit of a very special form :

$$
\hat{I}=I_{0} \exp \frac{I}{2} \int^{t}\left(\frac{I}{R_{g} C}-\frac{R}{L}\right) d t^{I}
$$

The special case of the steady state is of course included: $\hat{I}$ is constant for a "perfect match"

$$
\frac{I}{R_{g} C}=\frac{R}{I}
$$

between generator and circuit.

For a given experiment all circuit elements and the program for $\hat{I}$ and $\hat{V}_{g}$ are prescribed. The only parameter that is still free is impedance $\boldsymbol{R}_{\mathrm{g}}$ of the generator.

One may wish to maximize the energy transfer at the end of the heating when the power has reached its maximum. That is, we require $\mathrm{P}=\mathrm{P}_{\max }$ for $t=\mathrm{t}_{2}$. According to (29) this is possible if and only if

$$
\frac{I}{\mathrm{R}_{\mathrm{g}} \mathrm{C}}=\left(\frac{\mathrm{R}}{\mathrm{L}}+2 \frac{\dot{I}}{\mathrm{I}}\right)_{t=t_{2}}
$$


The eenerator impedance must be larger than for a "perfect match" to the resistance $\mathrm{R}\left(\mathrm{t}_{2}\right)$. Using the formulas (19.3), (20.3), (19.4) and (20.4), the reference values and the normalized (non dimensional) field strength, this may be written:

$$
R_{g} C=\frac{a_{0}}{v_{0}}\left(2+k_{\theta, z}\right) r_{I}(\beta)
$$

where

$$
\begin{aligned}
& r_{1}(\beta)=\beta^{-3 / 2}+\beta^{1 / 3} \\
& k_{\theta}^{-1}=\frac{2}{3}\left(b^{2} / a_{0}^{2}-1\right) \\
& k_{z}^{-1}=\frac{4}{3} \lg \left(b / a_{0}\right)
\end{aligned}
$$

In other cases one may wish to maximize the efficiency, $\boldsymbol{\eta}_{\mathbf{1}}$ of energy transfer with respect to $\mathbb{R}_{\mathrm{g}}$ :

$$
\eta=\int_{0}^{t_{2}} \operatorname{Pdt}^{1} / \int_{0}^{t_{2}} P_{\max } d t^{1}
$$

Maximizing $\eta$ is not only desirable for the sake of energy economy; some generators 3) do not tolerate large reflections. They work well only if the efficiency is close to one. The result of this maximization can be presented most concisely by first defining six definite integrals :
$A=\int_{0}^{t} \dot{I}^{2} d t$
$B=\int_{0}^{t} I \dot{I} d t$
$c=\int_{0}^{t} \frac{R}{L} \operatorname{II} d t$

3) R. Keller, Helv. Phys. Acta 28, 328 (1965) 
$D=\int_{0}^{t_{2}} I^{2} d t \quad E=\int_{0}^{t^{2}} \frac{R}{L} I^{2} d t \quad F=\int_{0}^{t} \frac{R^{2}}{I^{2}} I^{2} d t$

These constants are fixed once the amplitude program is prescribed. Thus

$$
\begin{aligned}
& \frac{1}{L} \int_{0}^{t_{2}} P_{\text {max }} d t=R_{g} C(A+C+F / 4)+B+E / 2+D / 4 R_{g} C \\
& \frac{I}{I} \int_{0}^{t_{g}^{2}} P d t=2 B+E
\end{aligned}
$$

The maximum value of $\eta$ is obtained for

$$
\mathbb{R}_{g} C=\frac{1}{2}\left(\frac{D}{A+C+F / 4}\right)^{1 / 2}
$$

and becomes

$$
\eta_{\max }=\frac{2 B+E}{B+E / 2+\sqrt{D(A+C+F / 4)}}
$$

The amplitude programs which are useful for the production of pinches yield usually efficiencies above $90 \%$. The efficiency of energy transfer from generator to plasma becomes now according to (26)

$$
\eta_{p}=\frac{3 \delta_{p}}{3 s_{p}+4 \delta_{B}} \eta_{\max }
$$

We shall now apply the maximization procedure to the amplitude program (16). We assume that the heating starts at $T=0$. This restriction is of no practical consequence, but it allows us to give efficiency $\eta_{\max }$ and generator impedance values $\boldsymbol{R}_{g}$ as 
functions of only one variable, the final normalized field strength $\beta$. Using formulas (34), (35), (36) together with (15) and (16) and keeping in mind that $I$ is proportional to $\beta$, one obtains without much trouble:

$$
\begin{aligned}
& \eta_{\max }=\frac{2}{1+\left(f_{2} f_{3}\right)^{1 / 2} / f_{1}} \\
& R_{g} C=\frac{a_{0}}{v_{0}}\left(2+k_{\theta z}\right)^{-1} r_{2}(\beta)
\end{aligned}
$$

The functions $f_{i}(\beta)$ are defined by

$$
\begin{aligned}
& f_{1}=\frac{1}{2} \beta^{2} \\
& f_{2}=2 \beta^{1 / 2}+\frac{3}{7} \beta^{7 / 3} \\
& f_{3}=\int_{0}^{\beta} \frac{\beta^{2} d \beta}{\beta^{-1 / 2}+\beta^{4 / 3}}
\end{aligned}
$$

and

$$
r_{2}(\beta)=\left(f_{3} / f_{2}\right)^{1 / 2}
$$

Fig. 4 shows $\eta_{\max }(\beta), r_{1}(\beta)$ and $r_{2}(\beta)$ as functions of $\beta$. 
Summary

The determination of the design parameters for a particular experiment is easily accomplished by means of the various graphs. One can begin by fixing the density $n_{0}$ and the radius $a_{0}$ of the constricted plasma column as well as the applied frequency $f$. From Fig. I the reference values $B_{0}, T_{0}, v_{0}^{-1}$ and $Q_{0}$ are then read off. They indicate the field, temperature, "heating velocity", and power at the transition. Next one decides what temperature $\mathrm{T}_{f}$ one wishes to reach and this gives the final value of $\beta=\left(\mathrm{T}_{\mathrm{f}} / \mathrm{T}_{\mathrm{O}}\right)^{1 / 2}$. Fig. 2 now gives the necessary heating time (15)

$$
t=\frac{a_{0}}{v_{0}} \tau(\beta)
$$

as well as the amplitude program and the maximum power (17)

$$
Q=q_{0} l a p(\beta)
$$

Finally, from $\mathbb{P i g} \cdot 4$ one can read off the values $r_{i}(\beta)$ which yield according to (30) and (37)

$$
\mathrm{R}_{\mathrm{g}} \mathrm{C}=r_{i} \frac{\mathrm{a}_{0}}{\mathrm{v}_{0}}\left(2+\mathrm{k}_{\theta z}\right)^{-1}
$$

The geometrical quantities $k_{\theta}$ and $k_{z}$ are given by (32) and (33). Using $r_{1}$ one utilizes best the peak power of the generator. Using $r_{2}$ one maximizes overall energy transfer and $?$ (Fig. 4) gives the efficiency.

Acknowledgement

The authors wish to thank M. Roux for his help with numerical calculations. 
Captions to Figures

Fig. I The reference values as functions of frequency and density. The units are : $f$ in mega cycles, $n$ in $10^{21}$ meter $^{-3}, q_{0}$ in mega watt / meter.centimeter, $\mathrm{B}_{0}$ in gauss, $\mathrm{v}_{0}$ in centimeter/microsecond, $\mathrm{T}_{0}$ in $10^{4}$ degrees Kelvin.

Fig. 2 The standard amplitude program : normalized field strength $\beta$ versus normalized time $\tau$ and the normalized plasma heating power $p$ as a function of $\beta$.

Fig. 3 The circuit coupling the generator to the plasma.

Fig. 4 The normalized generator impedance $r_{1}$ for maximizing peak power, $r_{2}$ for maximizing total energy transfer, as functions of normalized final field strength $\beta$, and the efficiency of energy transfer, $\eta$, for the second case. 


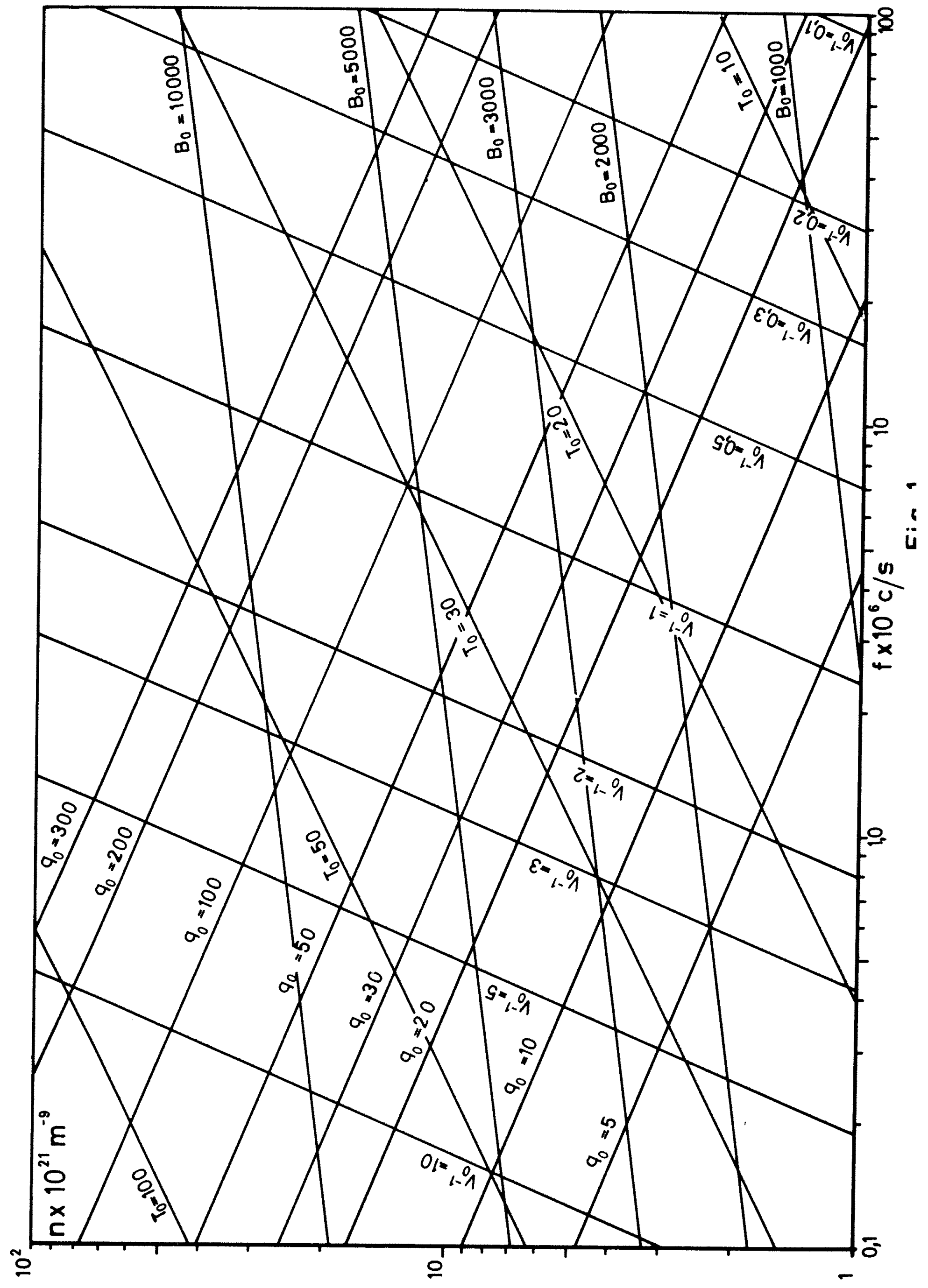




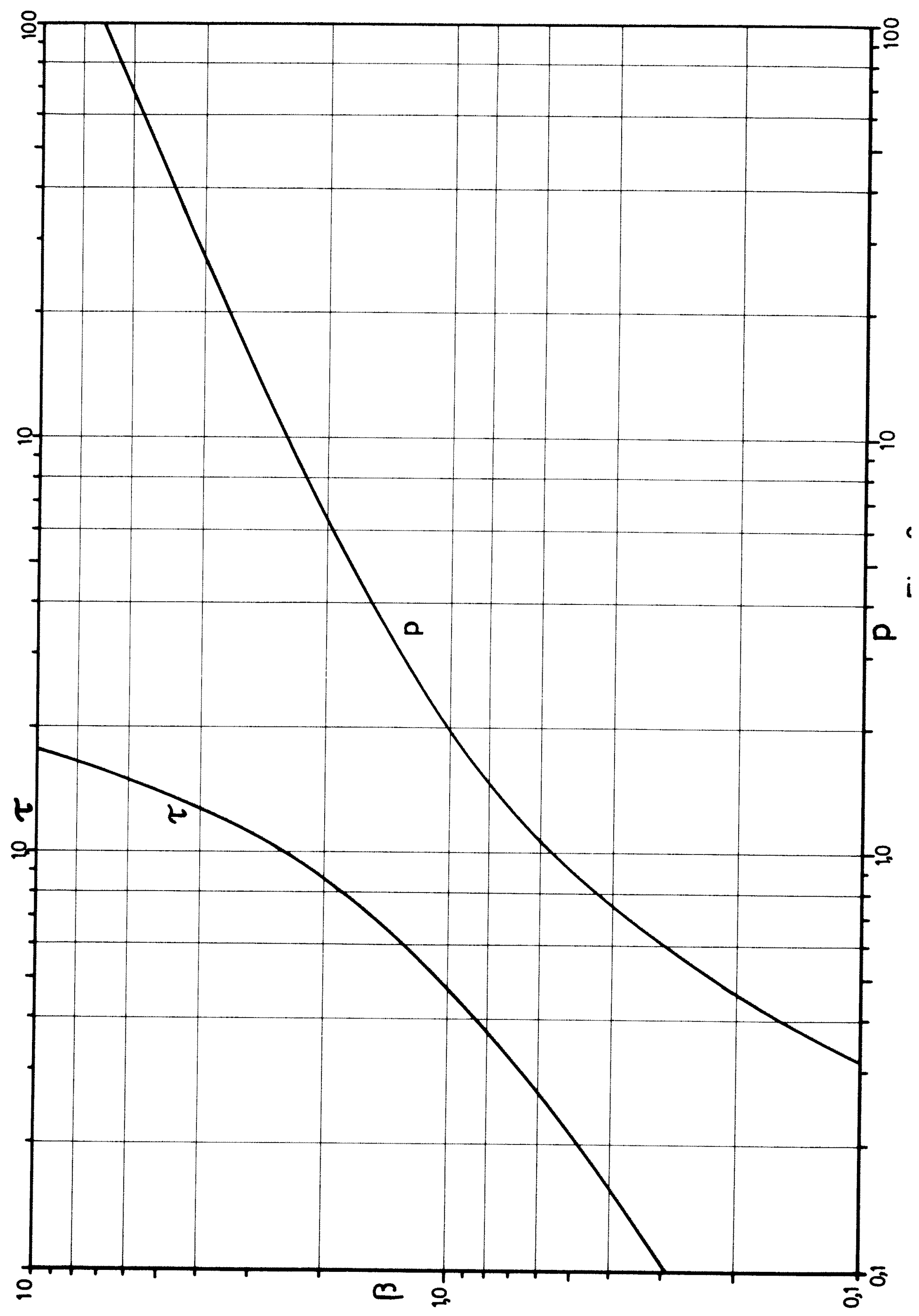




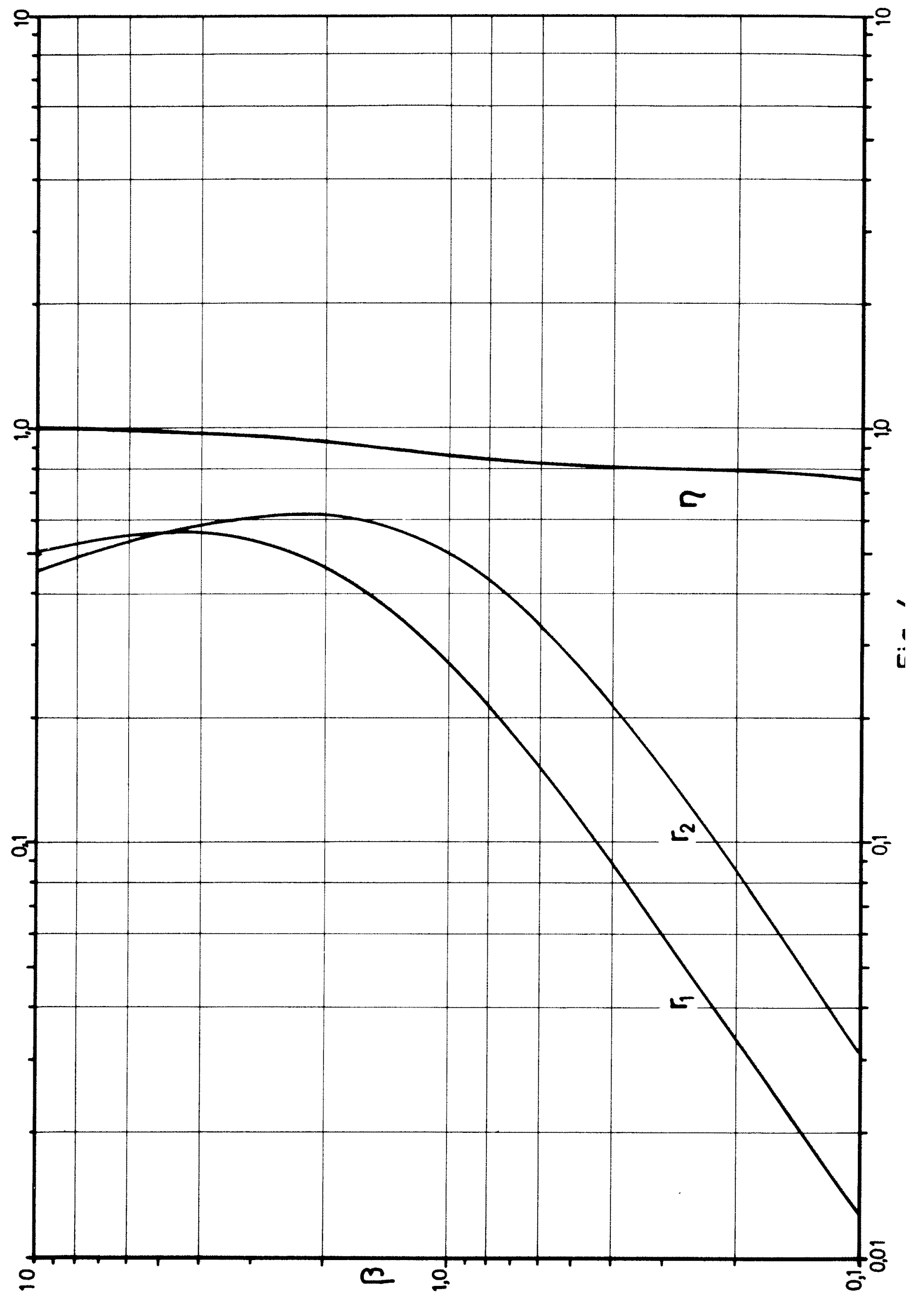

